\title{
MÉTODOS ÁGEIS SOB A ÓTICA DA INFORMAÇÃO
}

\section{MÉTODOS ÁGILES DESDE EL PUNTO DE VISTA DE LA INFORMACIÓN}

\author{
Eder Junior Alves* \\ Carlos Alberto Gonçalves** \\ Marcello Peixoto Bax***
}

\begin{abstract}
RESUMO
Introdução: Desde que Paul M. G. Otlet evidenciou o termo documentação em 1934, propondo como coletar e organizar o conhecimento do mundo, muitas pesquisas científicas direcionaram observações para o estudo da Ciência da Informação. Métodos e técnicas surgiram com uma visão de mundo sob o prisma da informação. Os métodos ágeis seguem esta tendência.
\end{abstract}

Objetivo: O propósito é analisar a relevância do fluxo de informação para as organizações que adotam os métodos ágeis, entendendo como a inovação de processo recebe influência desta prática.

Metodologia: Trata-se de um estudo bibliométrico com fundamentos da Revisão Sistemática de Literatura (RSL). A integração entre a técnica RSL interagindo com a ferramenta Summarize é uma nova proposta metodológica.

Resultados: O método ágil Scrum aparece com maior número de publicações no SPELL. Em comparação, os resultados das publicações do Google Scholar apontaram para a importância das práticas e comportamentos das equipes. No repositório DirectScience, fatores críticos de sucesso em gerenciamento de projetos e desenvolvimento de software aparecem em destaque.

Conclusões: Ficou evidente que os métodos ágeis estão sendo utilizados como inovações de processos. Os benefícios e vantagens evidenciam-se com o maior fluxo de informações interno e externo, entre equipes e o cliente. Por sua prevalência na literatura, o Scrum merece atenção por parte das firmas.

Palavras-chave: Métodos ágeis. Inovação de processo. Fluxo de informação. Revisão sistemática de literatura.

*Doutorando em Administração de Empresas. E-mail: ederjr@yahoo.com

** Doutor em Administração de Empresas. E-mail: carlos@face.ufmg.br

***Doutor em Ciência da Informação. E-mail: bax.ufmg@gmail.com 


\section{INTRODUÇÃO}

O objetivo desta Revisão Sistemática de Literatura (RSL) é avaliar a relevância do fluxo de informação para as organizações que adotam a metodologia ágil, atuando dentro da economia digital. A criação do conhecimento será levada em consideração ao observar a influência da comunicação entre as partes interessadas, bem como, a conexão entre métodos ágeis e a inovação de processos. A abordagem holística criada por Takeuchi e Nonaka (1986) sugere a formação integradora entre instabilidade embutida, aprendizado diversificado, auto-organização de equipes de projetos, fases de desenvolvimento sobrepostas, controle sútil e transferência organizacional do aprendizado para formar uma rápida e flexível maneira de desenvolver novos produtos. Esta estrutura pode atuar como veículo para introdução de ideias criativas, orientadas para o mercado e processos nas organizações.

Segundo Choo (1998), a informação é um recurso estratégico das firmas, sendo muito mais do que apenas outro fator de produção. Nesta perspectiva, Le Codiac (1996) estudou o papel e a importância da necessidade de informação para o indivíduo, o autor afirma que informação será transformada em conhecimento. De acordo com Saracevic (1999), esta relação em que a informação é uma característica dominante na construção do conhecimento fica evidente na atualidade.

A busca por agilidade se torna inevitável. A partir da crise do software na década de 1970 inicia-se a utilização do modelo tradicional denominado sequencial linear ou cascata para realizar o gerenciamento dos projetos. "A medida que a importância do software cresce, busca-se desenvolver tecnologias que tornem mais fácil, mais rápido e menos dispendioso construir e manter programas de computadores de alta qualidade." Pressman (2006, p.2).

Larman (2003) e Mnkandla (2008) comentaram que a abordagem em cascata do modelo tradicional, com foco no pensamento sequencial em que as atividades são feitas para produzir um resultado e a premissa de que os membros da equipe não precisam aprender nada torna-se limitada. O Agile 
Manifesto (2001) reúne os princípios para a nova abordagem. Boehm e Turner (2004) e Fowler (2002) mencionaram que as metodologias ágeis emergem com foco centralizado em pessoas ao invés de processos, reduzindo a quantidade de documentos desnecessários.

Neste contexto, o problema de pesquisa aqui apresentado, em forma de questão, é saber quais são os principais métodos ágeis e sua influência no gerenciamento de projetos e na perspectiva das publicações cientificas no Brasil? Este trabalho justifica-se por analisar e identificar o vínculo existente entre os métodos de gerenciamento ágeis com a gestão da informação e do conhecimento. Assim, optou-se pela RSL para obter medidas em termos bibliométricos.

Em suma, até o momento, apresentou-se as principais tendências atuais vinculadas aos temas da pesquisa e os conceitos que serão trabalhados ao longo do texto. O objetivo do artigo e o problema da pesquisa foram declarados, clemenciando solução para a questão relacionada com este trabalho acadêmico.

\section{REVISÃO SISTEMÁTICA DA LITERATURA}

A RSL é um método para realizar estudo bibliométrico. O foco é na objetividade e conhecimento de outros autores que são mencionados ou referenciados. De acordo com Kitchenham (2004), existem três fases principais: planejamento, condução e reporte dos resultados. Segundo Littell, Corcoran e Pillai (2008), a RSL busca localizar e sintetizar pesquisas que dizem respeito a uma questão particular, usando procedimentos organizados, transparentes e replicáveis em cada passo do processo. De acordo com os autores, não é o mesmo que a meta-análise. A meta-análise é um conjunto de métodos estatísticos para combinar resultados quantitativos advindos de múltiplos estudos para produzir um resumo do conhecimento empírico de um determinado tópico.

Por outro lado, ao olhar para a disciplina Ciência da Informação, nota-se o caráter autônomo e interdisciplinar característico dos trabalhos científicos 
deste campo acadêmico. Barreto (1994) vinculou o uso das informações para satisfazer necessidades humanas. Segundo Saracevic (1999), existem preocupações que recaem na perda da visão do lado dos usuários (dos seres humanos). Com estas perspectivas, esta linha de pesquisa busca olhar e compreender o fenômeno informacional. Entre os temas de interesse estão o desenvolvimento científico e o uso da informação. Estas buscas remetem à cienciometria, infometria, webometria e a bibliometria.

Assim, este trabalho bibliométrico pesquisou e analisou fontes secundárias com direcionamento para temas da Ciência da Informação e do Gerenciamento de Projetos no desenvolvimento de software, entre elas, informação, agilidade e projetos. Como resultado desta análise, foi possível fornecer evidências dos benefícios e limitações dos métodos ágeis para estes construtos.

A necessidade desta RSL concentra-se na busca por informações existentes sobre a influência dos métodos ágeis para a Ciência da Informação. O ponto de início para definir o protocolo de revisão, vai além de saber como as metodologias de gerenciamento ágeis vem sendo usadas como inovações de processos, pergunta inicial vinculada ao problema. Acrescenta-se a seguinte pergunta em função de revisões prévias, qual o vínculo dos métodos ágeis com a Informação?

O primeiro critério da RSL foca nos resultados de estudos que usam o fluxo de informação e os métodos de gerenciamento de projetos ágeis para desenvolver conhecimento empresarial. Este direcionamento estabelece um conjunto de termos que são relevantes nos principais documentos do escopo adotado. O critério utilizado nos títulos dos documentos no contexto brasileiro foram agile, information, informação, project e projeto. Também foram feitas as combinações agile e information, agile e informação e agile e projeto.

O segundo critério levou em conta leituras prévias de teses e artigos a respeito dos métodos ágeis, bem como resultados fruto da pesquisa do primeiro critério. Trata-se de utilizar os dez métodos ágeis como palavraschave, são eles, Adaptative Software Development (ASD), Crystal, Feature Driven Development (FDD), Agile Model Driven Development (AMDD), ICONIX, 
Scrum, Dynamic System Development Methodology (DSDM), Lean Development, eXtreme Programming e Kanban.

A base de dados dos documentos analisados no contexto brasileiro foi o Scientific Periodicals Electronic Library (SPELL). Em relação ao contexto global optou-se por Google Scholar e ScienceDirect.

A estratégia de buscar nas bases de dados fora do Brasil foi menor em número de palavras-chave. Apenas uma palavra foi utilizada para extrair os artigos. Esta palavra foi Agile e a estratégia foi consultar apenas os títulos dos documentos. A opção pelo Google Scholar e ScienceDirect está relacionada as limitações de acesso e facilidade na obtenção dos documentos. Estas limitações estão relacionadas com a necessidade de pagamento financeiro pela obtenção dos artigos em outras bases. Assim, optou-se por estas gratuitas.

\subsection{Scientific Periodicals Electronic Library}

A Tabela 1 mostra os resultados ao pesquisar as palavras-chave designadas no protocolo como primeiro critério de consulta. Utilizou-se o campo para títulos ao selecionar os artigos extraídos na base de dados SPELL.

Tabela 1: Consulta no SPELL pelo título do artigo

\begin{tabular}{lccc}
\hline $\begin{array}{c}\text { Palavras- } \\
\text { chave }\end{array}$ & $\begin{array}{c}\text { Quantidade } \\
\text { de artigos }\end{array}$ & $\begin{array}{c}\text { Periódico com maior } \\
\text { número de artigos }\end{array}$ & $\begin{array}{c}\text { Autor com maior número de } \\
\text { publicações }\end{array}$ \\
\hline Agile & 9 & $\begin{array}{c}\text { Revista de Gestão e } \\
\text { Projetos (4) }\end{array}$ & $\begin{array}{c}\text { Empate com uma publicação para } \\
\text { cada autor }\end{array}$ \\
\hline Information & 742 & $\begin{array}{c}\text { Journal of Information } \\
\text { Systems and } \\
\text { Technology } \\
\text { Management (106) }\end{array}$ & Edson Luiz Riccio (18 publicações) \\
\hline Informação & 713 & $\begin{array}{c}\text { Perspectivas em } \\
\text { Gestão \& } \\
\text { Conhecimento (52) }\end{array}$ & Edson Luiz Riccio (14 publicações) \\
\hline agile e \\
information & 1 & $\begin{array}{c}\text { Journal of Information } \\
\text { Systems and } \\
\text { Technology } \\
\text { Management (1) }\end{array}$ & Empate com uma publicação para \\
cada autor
\end{tabular}


Eder Junior Alves, Carlos Alberto Gonçalves, Marcello Peixoto Bax Métodos ágeis sob a ótica da informação

\begin{tabular}{lccc}
\hline Projeto & 500 & $\begin{array}{c}\text { Revista de Gestão e } \\
\text { Projetos (100) }\end{array}$ & Roberto Sbragia (10 publicações) \\
\hline $\begin{array}{c}\text { agile e } \\
\text { projeto }\end{array}$ & 3 & $\begin{array}{c}\text { Revista de Gestão e } \\
\text { Projetos (3) }\end{array}$ & $\begin{array}{c}\text { Empate com uma publicação para } \\
\text { cada autor }\end{array}$ \\
\hline
\end{tabular}

Fonte: Elaborado pelos autores.

Esta Tabela 1 apresenta a quantidade de artigos publicados, os periódicos que publicaram o maior número de artigos e os autores que tiveram o maior número de publicações. Quando houve empate, os autores não foram mencionados. Os resultados das métricas foram em relação as palavras-chave da primeira coluna. Isso quer dizer que não há vínculo entre as outras colunas.

Percebeu-se que nenhum autor publicou mais de uma vez em função das palavras-chave com foco em métodos ágeis. A quantidade de artigos que foram encontrados com foco exclusivo na palavra Agile foram nove, sendo que a revista que mais publicou foi a Revista de Gestão e Projetos (GeP). Trata-se de uma revista que possui um foco mais ativo em Gerenciamento de Projetos e temas vinculados.

O número de publicações em métodos ágeis no Brasil é muito pequeno se comparado com os temas desenvolvidos para a Ciência da Informação. Nota-se que são 742 artigos retornados com a palavra Information em comparação aos nove artigos com a palavra Agile.

Outra consulta realizada no SPELL levou em conta o segundo critério do protocolo para os nomes dos métodos ágeis definidos. A Tabela 2 a seguir mostra os resultados encontrados na consulta aos resumos do SPELL. Devido aos resumos não apresentarem abreviações utilizou-se apenas os nomes dos métodos ágeis com o operador 'e' para obter a interseção.

Tabela 2: Consulta no SPELL pelo resumo do artigo, obtendo publicações em métodos ágeis

\begin{tabular}{lccc}
\hline $\begin{array}{c}\text { Método ágil como } \\
\text { palavra-chave }\end{array}$ & $\begin{array}{c}\text { Quantidade de } \\
\text { artigos relacionado } \\
\text { ao método }\end{array}$ & $\begin{array}{c}\text { Periódico com } \\
\text { maior número } \\
\text { de artigos }\end{array}$ & $\begin{array}{c}\text { Referências e } \\
\text { considerações sobre os } \\
\text { resultados }\end{array}$ \\
\hline $\begin{array}{l}\text { Adaptive Software } \\
\text { Development }\end{array}$ & 1 & - & Dolci (2011) \\
\hline Crystal & 3 & - & $\begin{array}{c}\text { Somente o artigo do autor } \\
\text { Dolci (2011) trata de } \\
\text { métodos ágeis }\end{array}$ \\
\hline Feature Driven & 1 & - & Dolci (2011) \\
\hline
\end{tabular}


Eder Junior Alves, Carlos Alberto Gonçalves, Marcello Peixoto Bax Métodos ágeis sob a ótica da informação

\begin{tabular}{|c|c|c|c|}
\hline \multicolumn{4}{|l|}{ Development } \\
\hline $\begin{array}{l}\text { Agile Model Driven } \\
\text { Development }\end{array}$ & 1 & - & $\begin{array}{c}\text { Não aparece com esse nome } \\
\text { em Dolci (2011) e sim como } \\
\text { Agile Modeling (AM) }\end{array}$ \\
\hline ICONIX & 0 & - & - \\
\hline Scrum & 7 & $\begin{array}{c}\text { Revista de Gestão e } \\
\text { Projetos (3) }\end{array}$ & $\begin{array}{c}\text { Silva e Neto (2015) } \\
\text { Carvalho, Barbosa e Silva } \\
(2014) \\
\text { Terlizzi e Biancolino (2014) } \\
\text { Negreiro e Barbosa (2013) } \\
\text { Vallerão e Roses }(2013) \\
\text { Dolci }(2011) \\
\text { Soares }(2004)\end{array}$ \\
\hline $\begin{array}{l}\text { Dynamic System } \\
\text { Development } \\
\text { Methodology }\end{array}$ & 1 & - & $\begin{array}{c}\text { Em Dolci (2011) aparece } \\
\text { como Dynamic Systems } \\
\text { Development Method } \\
\text { (DSDM) }\end{array}$ \\
\hline Lean Development & 1 & - & $\begin{array}{c}\text { O artigo refere-se a Lean } \\
\text { Thinking } \\
\text { Sellito, Borchardt e Pereira } \\
(2003)\end{array}$ \\
\hline eXtreme Programming & 2 & - & $\begin{array}{c}\text { Dolci }(2011) \\
\text { Soares }(2004)\end{array}$ \\
\hline Kanban & 5 & $\begin{array}{c}\text { Revista de } \\
\text { Administração (2) }\end{array}$ & $\begin{array}{l}\text { Jabbour et al. (2013) } \\
\text { Junior e Filho (2009) } \\
\text { Graeml e Csillag (2009) } \\
\text { Contador et al. (2005) } \\
\text { Vitorino e Terra (2002) }\end{array}$ \\
\hline
\end{tabular}

Fonte: Elaborado pelos autores.

Ocorreu que o método ágil Scrum foi o que teve maior número de citações. São sete artigos sendo que três foram publicados na Revista Gestão e Projetos (GeP). Percebe-se que a referência Dolci (2011) foi a que mais aparece nas publicações. Trata-se da mesma referência que leva em conta os métodos pesquisados. Ao ler este artigo percebe-se que é o único correlato desta bibliometria. Dolci (2011), avaliou os métodos ágeis eXtreme Programming (XP), Dynamic Systems Development Method (DSDM), Scrum,

${ }^{1}$ Lean Thinking é um método para pensar como organizar as atividades humanas na visão dos negócios, distribuindo benefícios para a sociedade e valor para os indivíduos ao mesmo tempo que elimina desperdícios. $O$ termo foi cunhado por James $P$. Womack e Daniel T. Jones buscando capturar os conceitos do Sistema Toyota de Produção. No caso do Lean Development o foco seria na aplicação em projetos. Parece haver semelhanças, entretanto a ênfase é outra. 
Crystal, Agile Modeling (AM) ou Agile Model Driven Development (AMDD), Feature Driven Design (FDD) e Adaptative Software Development (ASD) na perspectiva do desenvolvimento de software no cenário brasileiro utilizando estes métodos ágeis no processo de inovação.

Observa-se na Tabela 2 que o Kanban aparece em cinco publicações. $O$ exame detalhado destes artigos remete a uma esfera da gestão de operações e processos. Os resultados mostram que para ser utilizado em Gerenciamento de Projetos, o Scrum é o mais citado na literatura extraída. Sabe-se que no ambiente empresarial a junção do Scrum com o Kanban já é uma prática, entretanto, não se pode afirmar que os pesquisadores estão publicando ou se interessando pelo tema até o momento. O Lean Development não é vinculado aos métodos ágeis para gerenciamento de projetos, apesar do nome.

Resumindo, observa-se que existem diversas perspectivas envolvendo os métodos ágeis segundo os resultados do SPELL. Chua e Yang (2008) argumentaram sobre a necessidade de uma grade curricular na Ciência da Informação que tivesse uma abordagem multidisciplinar em sua essência. $O$ foco deveria, segundo os autores, ser na intersecção de diferentes paradigmas para possibilitar uma potencial visão com ângulos diferentes de problemas antigos, bem como abordagens sob novas perspectivas para novos problemas. Ora, desta forma afirma-se que este estudo é sobre esta relação. Com esta argumentação, outros livros e outras referências consideradas relevantes pelos pesquisadores para satisfazer o objetivo da pesquisa foram analisadas.

\subsection{Google Scholar}

O Tabela 3 a seguir mostra os resultados ao pesquisar nas métricas do Google Scholar com a palavra-chave Agile.

Tabela 3: Análise por título do artigo no Google Scholar

\begin{tabular}{ccc}
\hline Palavra-chave & Publicações & h5-index \\
\hline \multirow{2}{*}{ Agile } & Agile Conference & 13 \\
\cline { 2 - 3 } & AGILE Conf & 12 \\
\cline { 2 - 3 } & XP/Agile Universe & 11 \\
\hline
\end{tabular}

Fonte: Elaborado pelos autores. 
Segundo o site da Google, o h5-index é o índice h para artigos publicados nos últimos cinco anos completos. Trata-se do maior número h, sendo que $\mathrm{h}$ artigos publicados entre 2010 e 2014 ter pelo menos $\mathrm{h}$ citações cada. $\mathrm{O}$ indicador de índice $\mathrm{h}$ significa número de artigos (h) que tenham recebido pelo menos $\mathrm{h}$ citações.

Fazendo o refinamento das publicações na Agile Conference com índice h5 igual a treze, chega-se a Tabela 4 contendo os títulos, autores, número de citações e ano da publicação para cada artigo publicado nesta conferência.

O artigo com maior número de citações dentre estes treze é uma revisão sistemática da literatura existente com abordagem no design centrado no cliente e sua integração com o desenvolvimento de software ágil. Ocorreu interesse porque houve uma cooperação entre pesquisadores brasileiros e internacionais (Nova Zelândia e Canada) para a criação do documento. Silva et al. (2011) propõem a existência de um modelo comum nos processos em que os artefatos são utilizados para dar suporte na colaboração entre designers e desenvolvedores de software.

Tabela 4: Análise da frequência por referências publicadas na Agile Conference

\begin{tabular}{|c|c|c|c|}
\hline Título & Referência & Citado por & Ano \\
\hline $\begin{array}{l}\text { User-Centered Design and Agile Methods: } A \\
\text { Systematic Review }\end{array}$ & Silva et al. (2011) & 51 & 2011 \\
\hline $\begin{array}{l}\text { Understanding Post-Adoptive Agile Usage--An } \\
\text { Exploratory Cross-Case Analysis }\end{array}$ & $\begin{array}{c}\text { Senapathi e } \\
\text { Srinivasan (2011) }\end{array}$ & 27 & 2011 \\
\hline $\begin{array}{l}\text { An Iterative Approach for Development of } \\
\text { Safety-Critical Software and Safety Arguments }\end{array}$ & $\begin{array}{c}\text { Ge; Paige e } \\
\text { Mcdermid (2010) }\end{array}$ & 23 & 2010 \\
\hline Survey of Agile Tool Usage and Needs & $\begin{array}{l}\text { Azizyan, Magarian e } \\
\text { Kajko-Matsson } \\
(2011)\end{array}$ & 23 & 2011 \\
\hline $\begin{array}{l}\text { Using Factor Analysis to Generate Clusters of } \\
\text { Agile Practices (A Guide for Agile Process } \\
\text { Improvement) }\end{array}$ & $\begin{array}{l}\text { Abbas, Gravell e } \\
\text { Wills (2010) }\end{array}$ & 20 & 2010 \\
\hline $\begin{array}{l}\text { Why Scrum Works: A Case Study from an } \\
\text { Agile Distributed Project in Denmark and India }\end{array}$ & $\begin{array}{c}\text { Pries-Heje e Pries- } \\
\text { Heje (2011) }\end{array}$ & 19 & 2011 \\
\hline $\begin{array}{l}\text { Agile Development and User Experience } \\
\text { Design Integration as an Ongoing } \\
\text { Achievement in Practice }\end{array}$ & $\begin{array}{l}\text { Ferreira, Sharp e } \\
\text { Robinson (2012) }\end{array}$ & 17 & 2012 \\
\hline $\begin{array}{l}\text { Classes of Distributed Agile Development } \\
\text { Problems }\end{array}$ & $\begin{array}{c}\text { Kajko-Mattsson, } \\
\text { Azizyan e Magarian } \\
(2010)\end{array}$ & 16 & 2010 \\
\hline $\begin{array}{l}\text { Documentation Work in Agile Teams: The Role } \\
\text { of Documentation Formalism in Achieving a } \\
\text { Sustainable Practice }\end{array}$ & $\begin{array}{l}\text { Stettina, Heijstek e } \\
\text { Faegri (2012) }\end{array}$ & 15 & 2012 \\
\hline The Combination of Agile and Lean in & Wang (2011) & 15 & 2011 \\
\hline
\end{tabular}




\begin{tabular}{llll}
\hline $\begin{array}{l}\text { Software Development: An Experience Report } \\
\text { Analysis }\end{array}$ & & & \\
\hline $\begin{array}{l}\text { Analysis of Federated and Global Scheduling } \\
\text { for Parallel Real-Time Tasks }\end{array}$ & Li et al. (2014) & 14 & 2014 \\
\hline $\begin{array}{l}\text { Knowledge Management in Distributed Agile } \\
\text { Software Development }\end{array}$ & $\begin{array}{c}\text { Dorairaj, Noble e } \\
\text { Malik (2012a) }\end{array}$ & 14 & 2012 \\
\hline $\begin{array}{l}\text { Driving Process Improvement via Comparative } \\
\text { Agility Assessment }\end{array}$ & $\begin{array}{c}\text { Williams, Rubin e } \\
\text { Cohn (2010) }\end{array}$ & 14 & 2010 \\
\hline
\end{tabular}

Fonte: Elaborado pelos autores.

Ao aprofundar a análise nos artigos que estavam embutidos na publicação AGILE Conf da Tabela 3, observou-se que não continha relação com os métodos ágeis de gerenciamento. Analisando os documentos que estavam sob a publicação XP/Agile Universe obtêm-se a Tabela 5.

Ao baixar os arquivos referentes a estes artigos observou-se que estão reunidos em uma publicação em formato de livro. Esta acomodação pode ser a explicação para sua divulgação e destaque nas métricas do Google Scholar.

O artigo mais citado trata da dinâmica da equipe e os padrões de interação entre seus membros para determinar melhor desempenho. Segundo Dorairaj, Noble e Malik (2012b), o sucesso do desenvolvimento ágil de software depende da interação da equipe. Os autores realizaram um estudo baseado na Grounded Theory, que envolveu 40 profissionais ágeis de 24 empresas de software diferentes nos Estados Unidos da América (EUA), Índia e Austrália. Segundo eles, foram investigadas as principais preocupações das equipes ágeis distribuídas e os achados foram que as equipes ágeis dependem significativamente da interação.

A variação temporal dos documentos está entre o ano de 2010 e 2012. Em relação a variação espacial o contexto em que os estudos foram desenvolvidos abrange uma dimensão global.

Tabela 5: Análise das citações por referências publicadas na XP/Agile Universe

\begin{tabular}{lccc}
\hline Título & Referência & $\begin{array}{c}\text { Citado } \\
\text { por }\end{array}$ \\
\hline $\begin{array}{l}\text { Understanding Team Dynamics in Distributed Agile } \\
\text { Software Development. XP, 47-61 }\end{array}$ & $\begin{array}{c}\text { Dorairaj, Noble e Malik } \\
(2012 b)\end{array}$ & 23 \\
\hline Values and Assumptions Shaping Agile Development & $\begin{array}{c}\text { Ferreira, Sharp e } \\
\text { and User Experience Design in Practice. XP, 178-183 }\end{array}$ & $\begin{array}{l}19 \\
\text { Robinson (2010) }\end{array}$ \\
\hline Agile Undercover: When Customers Don't Collaborate. & Hoda, Noble e Marshall & 17 \\
\hline
\end{tabular}




\begin{tabular}{|c|c|c|}
\hline$X P, 73-87$ & $(2010)$ & \\
\hline $\begin{array}{l}\text { Understanding the Importance of Trust in Distributed } \\
\text { Agile Projects: A Practical Perspective. XP, 172-177 }\end{array}$ & $\begin{array}{l}\text { Dorairaj, Noble e Malik } \\
(2010)\end{array}$ & 16 \\
\hline $\begin{array}{l}\text { Towards Understanding Communication Structure in Pair } \\
\text { Programming. XP, 117-131 }\end{array}$ & Stapel et al. (2010) & 16 \\
\hline $\begin{array}{l}\text { Effective Communication in Distributed Agile Software } \\
\text { Development Teams. XP, 102-116 }\end{array}$ & $\begin{array}{l}\text { Dorairaj, Noble e Malik } \\
(2011)\end{array}$ & 15 \\
\hline $\begin{array}{l}\text { Agile Software Development Practice Adoption Survey. } \\
X P, 16-30\end{array}$ & $\begin{array}{c}\text { Kurapati, Manyam e } \\
\text { Petersen (2012) }\end{array}$ & 14 \\
\hline $\begin{array}{l}\text { Empirical Investigation on Agile Methods Usage: Issues } \\
\text { Identified from Early Adopters in Malaysia. XP, 192-207 }\end{array}$ & $\begin{array}{l}\text { Asnawi, Gravell e Wills } \\
\text { (2011) }\end{array}$ & 13 \\
\hline $\begin{array}{l}\text { Supporting Self-Organizing Agile Teams-What's Senior } \\
\text { Management Got to Do with It? XP, 73-87 }\end{array}$ & $\begin{array}{l}\text { Hoda, Noble e Marshall } \\
(2011)\end{array}$ & 13 \\
\hline $\begin{array}{l}\text { Simulating Kanban and Scrum vs. Waterfall with System } \\
\text { Dynamics. XP, 117-131 }\end{array}$ & Cocco et al. (2011) & 12 \\
\hline $\begin{array}{l}\text { Extreme Product Line Engineering-Refactoring for } \\
\text { Variability: } A \text { Test-Driven Approach. } X P, 43-57\end{array}$ & $\begin{array}{l}\text { Ghanam e Maurer } \\
(2010)\end{array}$ & 11 \\
\hline
\end{tabular}

Fonte: Elaborado pelo autor.

De acordo com Littell, Corcoran e Pillai (2008), o cuidado com a linguagem e interpretação dos resumos dos resultados é uma conduta a ser realizada na RSL. Resumos verdadeiros e acessíveis melhoram a utilização de uma revisão. Nesta linha, os pesquisadores desta pesquisa optaram por utilizar a ferramenta denominada Summarize. Trata-se de um engine disponibilizado online na Internet pela Tools4Noobs (2015). O foco não é entender como o algoritmo do Summarize realiza o processamento e sim analisar os resultados. Esta ferramenta fornece a relevância das sentenças e mostra as melhores palavras em função da pontuação. Os seguintes procedimentos foram realizados:

- Foram baixados os arquivos das Tabelas 4 e 5;

- Copiou-se todos os resumos em um arquivo do MS Word;

- A ferramenta Summarize foi acessada e todos os abstracts foram copiados, logo em seguida, executou para obter os resultados.

Os resultados fornecidos pelo Summarize como melhores palavras encontradas nos resumos dos artigos da Tabela 4 e Tabela 5 foram:

i. agile (pontuação: 77$)$

ii. software (pontuação: 39) 

iii. development (pontuação: 32)
iv. practices (pontuação: 24)
v. teams (pontuação: 23)
vi. distributed (pontuação: 17)
vii. paper (pontuação: 17)
viii. results (pontuação: 16$)$
ix. study (pontuação: 15)
x. team (pontuação: 15)

A palavra agile naturalmente apareceria em primeiro. Software e desenvolvimento mostram-se em seguida, fornecendo uma pista de que o setor de desenvolvimento de software é o segmento que mais utiliza os métodos ágeis. Práticas e equipes chamam a atenção. As categorias que serão estudas nesse trabalho estão alinhadas com esta perspectiva. Os papeis e responsabilidades das equipes, bem como, as técnicas e ferramentas utilizadas no trabalho diário de equipes desenvolvendo ou integrando sistemas de tecnologia da informação.

\subsection{Science Direct}

$\mathrm{Na}$ base de dados ScienceDirect, o critério da busca dos artigos foi somente a palavra Agile como palavra-chave no título. A busca foi fechada para retornar artigos em periódicos. Obteve-se 107 resultados. Ao incluir todos os resumos destes artigos na ferramenta Summarize as melhores palavras consideradas foram:

i. $\quad$ agile (pontuação 397);

ii. desenvolvimento (pontuação 215);

iii. software (pontuação 207);

iv. projeto (pontuação 104);

v. estudo (pontuação 88);

vi. resultados (pontuação 83);

vii. métodos (pontuação 80); 
viii. paper que foi traduzido para artigo (pontuação 77);

ix. $\quad$ método (pontuação 73);

$x$. projetos (pontuação 71 ).

Observa-se a relação que pode ser deduzida entre os métodos ágeis no desenvolvimento de projetos de software. Trata-se da principal justificativa para realizar uma revisão sistemática em profundidade dos construtos envolvidos.

Outra saída que o Summarize fornece são resumos a respeito dos textos incluídos. Tem-se em conta sua relevância perante o conjunto heterogêneo de artigos devido ao critério de utilizar a palavra Agile. A seguir são fornecidos os cinco primeiros resultados em relação aos 107 artigos. Trata-se de um resumo dos 107 abstracts (resumos dos artigos):

I. "[...] como são os objetivos das equipes para iterações diferentes do triângulo dourado dos fatores de sucesso de gerenciamento de projetos? O objetivo deste estudo é identificar os objetivos da iteração e as decisões críticas que se relacionam com o triângulo dourado dos fatores de sucesso de gerenciamento de projetos em equipes ágeis de desenvolvimento de software que trabalham em iterações de duas semanas", com pontuação de 1376;

II. "O objetivo deste artigo é explicar o método empírico e os resultados de análises e comparação do desempenho do desenvolvimento, qualidade do produto de processo incremental e processo Agile adaptado em dois projetos de um de tamanho médio, empresa de desenvolvimento de software de telecomunicações sistemáticas", com pontuação de 1328;

III. "[...] o objetivo deste estudo é usar/compartilhar a teoria dos modelos mentais como uma lente para examinar as práticas de metodologias ágeis de software para entender como as práticas ágeis de desenvolvimento de software permitem que as equipes para trabalhar em conjunto para concluir tarefas e trabalhar em conjunto de forma eficaz como uma equipe", pontuação de 1314; 
IV. "Conclusão desta pesquisa tem contribuído para o desenvolvimento de software e gerenciamento de projetos de literatura, examinando os objetivos da iteração em equipes ágeis e como eles se relacionam com o triângulo dourado dos fatores de sucesso de gerenciamento de projetos para ver se essas equipes incorporar os fatores triângulo dourado em seus objetivos e se eles incluem objetivos adicionais em suas iterações", pontuação de 1311 ;

V. "A fim de resolver esta questão, realizamos um estudo de caso dentro de um projeto de desenvolvimento ágil de software distribuído globalmente focado em utilizar o conceito de resíduos como uma lente para a identificação de não-valor produzir elementos de comunicação", pontuação de 1275.

Em termos de teorias e metodologias de pesquisa acadêmica nas Ciências Sociais Aplicadas, os pesquisadores notaram a presença nos artigos dos métodos empíricos, teoria dos modelos mentais, estudos de caso e a Grounded Theory.

Em suma, ao analisar estas bases de dados percebe-se que existe uma relação próxima entre os métodos ágeis e o gerenciamento de projetos de desenvolvimento de software. Percebe-se, ainda, o vínculo e foco destes métodos na iteração, papéis e responsabilidades entre as equipes. As práticas que são realizadas durante o período em que o processo de transformação ágil está sendo desenvolvido é um ponto de atenção, bem como, as ferramentas que são utilizadas pelas pessoas atuando nos projetos.

Não menos importante enfatizar a possibilidade ontológica que surge das discussões entre os autores durante a trabalhosa tarefa de criar e analisar por meio da RSL. Ontologia no sentido que foi proposta por Almeida (2014, p.244), segundo o autor "existe consenso de que o estudo da ontologia diz respeito aos tipos de coisas que existem". Em outras palavras, como já mencionado pelo autor e utilizado por Aristóteles, seriam as categorias. $O$ autor mencionou que o mais importante seria entender a ontologia como uma teoria 
das categorias como um sistema estruturado em níveis hierárquicos, na forma de árvore invertida e com nível superior denominado entidade.

Mnkandla (2008), em sua tese de doutorado, revisou a lista de métodos identificados na contemporaneidade e utilizou oito categorias para analisar os métodos ágeis: filosofia metodológica, modelo, técnicas e ferramentas, escopo metodológico, saídas metodológicas (entregas), adaptação e experiência, produto metodológico e papeis e responsabilidades. Com base na RSL feita no SPELL, outras referências bibliográficas consideradas e nas "coisas que existem", o enfoque deste estudo será limitado em apenas duas categorias:

- Técnicas e ferramentas: identificação de técnicas e ferramentas utilizadas pela metodologia. Pode-se considerar que a metodologia não necessariamente especifica técnicas e ferramentas;

- Papéis e responsabilidades: refere-se à alocação de papeis por meio do qual a produção de software em uma equipe de desenvolvimento é realizada. Este elemento essencialmente esboça quem faz o que no projeto (ABRAHAMSSON et al., 2002).

\section{RESULTADOS OBTIDOS EM FUNÇÃO DAS CATEGORIAS}

Com base no referencial teórico e resultados da customização do software Summarize, propõe-se que estes novos métodos ágeis, visando pessoas e com apelo para a agilidade e criatividade que agreguem valor aos negócios, podem ser considerados na gestão de projetos, em especial no desenvolvimento de software. De acordo com Booch, Rumbaugh e Jacobson (2005), a agilidade tornou-se a palavra-chave quando se descreve um processo moderno. Uma equipe ágil é aquela rápida e capaz de responder apropriadamente a mudanças.

Segundo Abrahamsson et al. (2002), o conceito de "ágil" ainda não obteve consenso. Entretanto, os autores ressaltam o interesse que os métodos relacionados com essa característica têm despertado nos praticantes e, mais 
recente, na academia. De acordo com os autores, a introdução do método eXtreme Programming, conhecido como XP, é o ponto de partida para várias outras abordagens no desenvolvimento ágil de software. Os autores comentam sobre métodos que estão sendo inventados ou redescobertos, como Crystal, Feature Driven Development e Adaptive Software Development. Além desses quatro métodos, a literatura mostrou que existem outros quatro sendo utilizados no estado atual, trata-se dos métodos Scrum (SUTHERLAND, 1995), (SCHWABER, 1997), Rational Unified Process (KRUCHTEN, 1999), Dynamic Systems Development Method (STAPLETON, 1997) e o Open Source Software Development.

Conforme Conboy (2009), os métodos ágeis mais relevantes no ano 2009 eram eXtreme Programming, conhecido como XP (BECK, 1999), Dynamic Systems Development Method, Scrum (SCHWABER E BEEDLE, 2001), Crystal (COCKBURN, 2007), Agile Modeling (AMBLER, 2012) e Feature Driven Design.

Em 2008, nove métodos ágeis foram identificados por Mnkandla (2008) em contraposição ao encontrado por Abrahamsson et al. (2002). Mnkandla (2008) não utilizou o Open Source Software Development em sua pesquisa, contudo incluiu o ICONIX e Lean Development. O método Kanban foi acrescentado porque aparece com relevância em trabalhos acadêmicos, mídias sociais, congressos e associações locais produtivas de software, totalizando dez métodos ágeis analisados neste artigo. Nenhum artigo da base SPELL tratou o Kanban como método para gerenciamento de projeto. As subseções seguintes apresentam os dez métodos em função destas categorias escolhidas.

\section{1 $\quad$ Adaptive Software Development (ASD)}

Neste método ágil aplica-se a antiga técnica de sessões de Joint Application Development (JAD). Segundo Highsmith (2000), o planejamento é baseado em resultados que também são conhecidos como planejamento baseado em componentes em que os planos tomam por base um grupo de 
características ou entregas. Planilhas são usadas para o planejamento baseado em componentes. Segundo o autor, revisões de grupos de foco de clientes é uma técnica útil no planejamento desse método. Outra técnica é o time-boxing que consiste na configuração dos tempos de entregas fixados por projetos e ciclos interativos. A time-boxing concentra-se em focar e avaliar a missão do projeto.

De acordo com Mnkandla (2008), o método ASD não tem uma lista compreensiva de responsabilidades, porém menciona que o patrocinador executivo deverá ter responsabilidade geral sob o produto, participantes nas sessões de desenvolvimento conjunta de aplicações, desenvolvedor e cliente.

\subsection{Crystal}

Conforme Cockburn (2007), os métodos Crystal não prescrevem técnicas em particular, mas enfatizam o uso de qualquer uma que venha da experiência da equipe. No caso do método Crystal Clear é feita a listagem das seguintes ferramentas: gerenciamento de compilador, versionamento, configuração e printing whiteboards. Para o método Crystal Orange lista-se ferramentas de versionamento, programação de testes, comunicação, acompanhamento de projetos, desenho e medição de desempenho. Drivers de tela são necessários para testes de Graphical User Interface (GUI). Segundo o autor, existe menção de metodologia sintonizando técnicas e uso de workshops de reflexão.

Segundo Abrahamsson et al. (2002), o Crystal Clear tem apenas uma equipe e o Crystal Orange várias. O patrocinador financia o projeto e entregas da declaração da missão. O projetista sênior mantém a estrutura da equipe, implementa a metodologia e projeta o sistema. O projetista-programador cria esboços de telas, projeta esboços e notas, modelo de objeto comum, código fonte, sistema empacotado, código de migração e casos de testes. O usuário ajuda com os casos de uso e esboços de telas. De acordo com o autor, o restante dos papéis pode vir misturados ou em uma lista prévia. Especialista de negócios pode vir do patrocinador, usuário ou projetista sênior. Coordenador deve vir dos projetistas. $O$ testador deve vir dos projetistas e o escritor também 
deve ser escolhido entre os projetistas. Conforme Cockburn (2007), o Crystal Orange tem os seguintes papéis adicionais arranjados em equipes: planejamento de sistema, mentoring de projeto, arquitetura, tecnologia, funções, infraestrutura e equipes de teste externa.

\subsection{Feature Driven Development (FDD)}

Trata-se de uma abordagem adaptativa e ágil para desenvolvimento de sistemas. Este método enfatiza os aspectos da qualidade através do processo e inclusão frequente de entregas tangíveis durante o monitoramento do progresso do projeto. "O FDD consiste em cinco processos sequenciais e fornece métodos, técnicas e guias necessários aos interessados do projeto para entregar o sistema" Abrahamsson et al. (2002, P.47). Segundo os autores, o processo do FDD consiste em desenvolver um modelo geral, construir uma lista de características, planejar pelas características, projetar (ou design) por característica e desenvolver por essas características. Sendo que esta lista de característica é apresentada pela equipe de desenvolvimento com base em cada função de valor incluída no sistema.

Conforme Palmer e Felsing (2002), existem três grupos de papéis no FDD: papéis chave, de suporte e adicionais. O gerente de projetos é um administrador e líder financeiro. $O$ arquiteto chefe é um projetista chefe e executa workshops em sessões de design. O gerente de desenvolvimento executa diariamente atividades e resolve problemas no desenvolvimento. $O$ chefe de programação é um desenvolvedor experiente que participa das análises e design dos requisitos. $\mathrm{O}$ dono de classe projeta, testa códigos e documenta o sistema sob a supervisão do chefe de programação. $O$ especialista de domínio tem conhecimento de como vários requisitos poderão ser implementados. O gerente de domínio lidera os especialistas de domínio no que diz respeito a diferentes opiniões em relação aos requisitos. O gerente de versões controla o progresso de relatórios. $O$ advogado de linguagem é um especialista em uma linguagem especifica ou tecnologia. $O$ engenheiro de construção configura, mantém e executa o processo construído. O toolsmith cria pequenas ferramentas para o desenvolvimento. Administrador de sistema 
configura, gerencia, procura problemas nos servidores, estações de trabalho e ambientes de teste e desenvolvimento. $O$ testador verifica se os requisitos de sistema foram satisfeitos. $O$ implementador converte dados existentes para 0 formato de novos sistemas. O autor ainda propõe o papel do escritor que escreve a documentação do usuário.

\subsection{Agile Model Driven Development (AMDD)}

De acordo com Ambler (2012), nenhuma ferramenta específica é mencionada no AMDD, exceto que ao pensar no uso de ferramentas deverá ser levado em conta a escolha das mais simples.

Ao buscar compreender os papéis e responsabilidade do AMDD, fica evidente que a formação da equipe de vir da seleção entre os desenvolvedores e interessados presentes na organização. Segundo Ambler (2012), estas equipes devem ser compostas de desenvolvedores trabalhando duro e auto motivados. O que não fica claro é como motivá-las. De acordo com o autor, a modelagem deve ser feita por estas equipes em que cada um participa de forma colaborativa.

\section{$3.5 \quad I C O N I X$}

Conforme Mnkandla (2008), o ICONIX propõe a utilização de diagramas UML, casos de uso, técnicas de análise orientada a objetos e design. Segundo - autor devem ser utilizadas técnicas e ferramentas de prototipação, desenvolvimento incremental/interativo e tutoriais de treinamentos para adotar tal método ágil.

A proposta do ICONIX para os papéis e responsabilidade são os programadores escreverem o código, os treinadores ministram cursos para a equipe do projeto em análise orientada a objetos e design. Segundo Mnkandla (2008), deve ser proporcionado para a equipe treinamento no método ágil ICONIX. De acordo o autor, o mentor é um consultor em tempo integral no local de trabalho, o analista escreve casos de uso e o projetista altera casos de uso 
nos diagramas de design. Por fim, o usuário fornece requisitos e revisões no design.

\subsection{Scrum}

Em termos de técnicas e ferramentas, segundo Schwaber e Beedle (2001), no Scrum existe o jogo do planejamento. Este jogo é uma técnica útil que resulta em uma lista de requisitos priorizados denominados lista de backlog. Os autores mencionaram ainda o uso do sprint como uma técnica em que a lista de requisitos priorizados são desenvolvidos em um produto executável sem permitir que novas mudanças nos requisitos aconteçam dentro da interação de trinta dias. O scrum diário é outra técnica de projeto que fornece controle gerencial diariamente em função do conhecimento do que está acontecendo e requer solução dos impedimentos. Não existem ferramentas específicas mencionadas, contudo a maioria das ferramentas de gerenciamento de projetos poderiam ser úteis de acordo com os autores.

Conforme o guia da empresa VMEdu (2013), existem papéis ou funções essenciais e não essenciais no Scrum. Os indivíduos com um papel essencial são completamente comprometidos com o projeto e são responsáveis pelo sucesso de cada interação do projeto como os demais interessados. O Product Owner (Dono do Produto), Scrum Master (Mestre Scrum) e Scrum Team (Equipe Scrum) exercem essas funções e responsabilidade. Segundo o guia do corpo de conhecimento do Scrum (SBOK Guide), os papeis não essenciais incluem os stakeholders (interessados), Scrum Guidance Body (Corpo do Guia Scrum), vendedor, Chief Product Owner (Chefe Dono do Produto) e o Chief Scrum Master (Chefe Mestre Scrum).

Nesta categoria papéis e responsabilidades, o Mestre Scrum garante as práticas do método Scrum e os valores que serão seguidos até o final do projeto. O Dono do Produto gerencia o projeto, controla e torna visível a lista de backlog do produto. A equipe Scrum tem autoridade para decidir ações e se auto organizar em função de completar um sprint (ciclo de interação). Conforme Schwaber e Beedle (2001), os clientes participam nos itens do 
backlog do produto e a gerência toma a decisão final e participa da definição das metas e requisitos.

\subsection{Dynamic System Development Methodology (DSDM)}

De acordo com Stapleton (1997), o DSDM utiliza workshops para estudo de negócio como uma técnica que reúne os interessados e esclarece requisitos de negócios. Ao optar pelo método ágil DSDM em uma organização, é recomendado realizar um treinamento sobre o DSDM para a equipe e outros interessados. Conforme o autor, este conjunto de métodos agrupados na metodologia Dynamic System Development não especifica ferramentas, entretanto, fornece um ambiente de suporte ideal em que enuncia a necessidade de análises de requisitos, prototipação de sistemas, design, construção de testes e engenharia reversa.

Para a categoria papéis e responsabilidades, Stapleton (1997), menciona que no DSDM, os desenvolvedores e membros seniores da equipe são envolvidos no ciclo de desenvolvimento do projeto. Segundo o autor, o coordenador técnico define a arquitetura do sistema e garante a qualidade técnica do projeto, o usuário embaixador traz o conhecimento dos usuários para o projeto e relata o progresso do projeto para os outros usuários. Além desses, podem ser designados um visionário que tem a visão precisa dos objetivos do negócio do sistema e do projeto. Stapleton (1997) comenta que o patrocinador executivo tem autoridade e responsabilidade financeira sobre 0 projeto.

\subsection{Lean Development (LD)}

De acordo com Mnkandla (2008), o LD segue um processo de análise de valorização que é usado para provar o valor de cada artefato. Segundo autor, a entrega rápida do produto é outra, se assim pode ser considerada, técnica que ataca as mudanças nos requisitos, quanto mais rápido você entregar menos mudanças de requisitos ocorrerão. 
No método ágil LD nenhuma menção especifica de papéis e responsabilidades foi observada, exceto que O LD se destina aos Chief Executive Officers (CEOs) antes de ser implementado em uma organização. Conforme Highsmith (2000), os gerentes de projetos e clientes são mencionados. Para efeito inferencial, este estudo leva em conta que apesar de somente os papeis terem sido designados para os últimos dois perfis, não explicitando suas responsabilidades, pode-se deduzir que estas advêm do cargo ou papel proposto.

\section{9 eXtreme Programming (XP)}

A programação em par é a técnica fundamental que contribuiu para a popularidade do XP. Programação test-first significa que o sistema é testado como foi construído ao invés de esperar até o final do teste. Refactoring é a técnica que permite mudança no design. Outras técnicas são: clientes no local e dono coletivo do código. O XP não especifica ferramentas para programação. Segundo Mnkandla (2008), a decisão sob ferramentas é determinada de acordo com as necessidades do projeto.

No método ágil XP os programadores escrevem testes e códigos. Clientes escrevem estórias, teste funcionais e conjuntos de prioridades de implementação. Testadores ajudam clientes escreverem testes e executam os testes funcionais, mantendo ferramentas de teste. De acordo com Abrahamsson et al. (2002), o acompanhador do andamento do trabalho (tracker) fornece feedback sobre exatidão das estimativas e traçam o progresso das interações, o coach guia a equipe para seguir o processo XP, um consultor é um membro externo que guia a equipe para resolver problemas e o gerente toma decisões.

\subsection{Kanban}

O Kanban possui ferramentas com origem no conceito just-in-time que é um dos pilares da manufatura enxuta (Lean). Por meio de cartões de requisição e fornecimento, painel ou quadro de controle, supermercados e containers as 
informações a respeito do andamento do trabalho ficam à vista e as atividades são cadenciadas de acordo com o progresso e limite definidos. Promove 0 controle do número de trabalho em progresso e fornece a sequência de atividades que serão realizadas por ordem de prioridade.

Kanban, segundo Guimaraes e Falsarella (2008), pode ser considerado um sistema de puxar em que a retirada ou execução de uma atividade sucessora puxa a antecessora. Kanban pode ser traduzido como cartão visível e está relacionado com a metodologia de gestão à vista dos sistemas produtivos.

O método kanban não prescreve papéis e responsabilidades. Tais definições devem surgir com base na maturidade da empresa e entendimento do processo de desenvolvimento. De acordo com Peeters (2013), a experiência mostra que as pessoas não gostam quando as responsabilidades e os títulos delas são trocados. Esta é a razão para sugerir não fazer isso no início da implementação do kanban.

Observado os resultados da análise das categorias, segue a relação entre os métodos ágeis e os fundamentos e conceitos da Ciência da Informação.

\section{MÉTODOS ÁGEIS SOB A ÓTICA DA INFORMAÇÃO}

Segundo Laudon e Laudon (2014), o crescimento da Internet, globalização do comércio e ascensão das economias da informação têm remodelado o papel dos sistemas de informações nos negócios e gerenciamentos das organizações. Choo (1998) argumenta que uma organização inteligente é aquela que usa seus recursos e capacidades informacionais para transformar informação em conhecimento, utilizando este conhecimento para sustentar e aumentar seu desempenho no ambiente. Davenport e Prusak (2003) comentam, em termos de contexto, experiência e interpretação, que o papel das pessoas nas tecnologias do conhecimento é parte integrante do seu sucesso. Fatos que potencializam o aparecimento de novos fatores humanos e tecnológicos. 
No alicerce para obtenção de novos métodos, técnicas e ferramentas está a forma como a informação e o conhecimento serão organizados, direcionando para um horizonte assertivo na realização das estratégias de negócios das organizações. Davenport e Prusak (2003) argumentam que o tempo é uma dimensão chave para encontrar uma solução de gestão do conhecimento numa determinada aplicação empresarial. Dessa forma, a Ciência da Informação assume seu papel de protagonista, definindo, conceituando os fundamentos e práticas para análise, transmissão e uso da informação. É por meio da Ciência da Informação que os estudiosos e pesquisadores podem fornecer significância e relevância do conhecimento.

De acordo Stevenson (2009), as organizações podem ser pensadas como operações que agregam valor, sendo vistas como um sistema em que existem insumos ou entradas, entre elas informações, que são consumidas com a finalidade de alcançar uma variedade de objetivos. O autor menciona que finanças, contabilidade, tecnologia da informação e recursos humanos dão suporte e facilitam o funcionamento da cadeia de valor.

Assim, a escolha de um projeto organizacional passa pela forma como a empresa aprende, muda ou interage com o contexto ambiental para obter aprendizado e conhecimentos que levem a distanciar dos competidores. Para criar conhecimento por meio de uma espiral, Nonaka e Takeuchi (1995) partem do princípio que essa construção se faz por meio da interação entre os empregados da empresa. Nota-se a disseminação de novo conhecimento em forma de feedbacks. Em uma direção o fluxo de informações e em outra o de conhecimento. Sem o qual, a utilização das técnicas e ferramentas dos novos métodos ágeis não seriam possíveis.

$\mathrm{Na}$ definição de inovação do manual de Oslo da OECD (2005), uma inovação pode ser considerada como uma implementação de um novo processo ou novo método organizacional que acontece por meio de práticas. Tais métodos ágeis agregam valor ao negócio das organizações através de melhorias no gerenciamento de seus projetos, criando novas informações e conhecimentos. Sendo assim podem ser considerados inovações de processo pois criam novas maneiras de realizar as atividades, agregando valor ao 
negócio das organizações que os adotam. Nessa linha justifica-se fundamentar esses métodos com uma visão para as teorias da gestão da informação e do conhecimento. Pois, não seria possível implementar esses métodos sem os conceitos e fundamentos da Ciência da Informação.

Esta pesquisa bibliográfica, em parte, consistiu em sumarizar evidências existentes a respeito dos benefícios e limitações dos métodos ágeis no contexto das empresas gerenciando suas informações. Os métodos ágeis emergem com um foco centralizado em pessoas, descobrindo maneiras melhores de desenvolver software por meio da valorização dos indivíduos e interações ao invés de utilizar processos e ferramentas.

\section{CONCLUSÕES}

No contexto de publicações acadêmicas brasileiras o método ágil Scrum merece destaque, obtendo sete artigos relacionados com sua aplicação. $O$ Kanban alcançou cinco publicações dentro do SPELL, observando, contudo, que os artigos tratavam apenas da utilização como gestão à vista em função da programação puxada dos sistemas produtivos. Mantendo o vínculo histórico com o Sistema Toyota de Produção. Percebeu-se que a Revista Gestão e Projetos (GeP) é a que mais interesse tem para os temas relacionados com métodos ágeis.

Ficou evidente que os métodos ágeis estão sendo utilizados como inovações de processo e o fluxo de informações torna-se necessário para colocar as técnicas e ferramentas em prática.

Nesta perspectiva, o vínculo dos métodos ágeis com gerenciamento de projetos de software pode ser verdadeiro e novas pesquisas sobre os temas devem ser conduzidas. A princípio, observa-se o surgimento efetivo desta relação na proposta do Agile Project Management (APM).

Observando-se que existe o vínculo entre o APM e a necessidade de fluxo de informações, percebe-se que a gestão do conhecimento se torna uma necessidade para que as atividades inter-relacionadas dentro dos processos, sofrendo reflexões e melhorias que culminam em inovações. Assim, trata-se do 
vínculo com a Ciência da Informação em termos de gestão das informações e do conhecimento.

$\mathrm{Na}$ análise de publicações no Google Scholar, percebe-se o foco nas práticas e equipes ágeis. A forma diferente de propor os papeis e responsabilidades nos métodos ágeis são inovações para as equipes de projeto, culminando na agregação de valor no árduo trabalho das atividades do dia a dia.

$\mathrm{Na}$ revisão da literatura observou-se nos estudos correlatos a presença de métodos empíricos, teoria dos modelos mentais, estudos de caso e a Grounded Theory para estudar os temas envolvidos com os métodos ágeis. A contribuição deste trabalho para os métodos de pesquisa nas Ciências Sociais Aplicadas são os procedimentos adotados, sequenciando a Revisão Sistemática da Literatura e a obtenção de resultados por meio de compêndios realizados pela ferramenta Summarize.

\section{REFERÊNCIAS}

ABBAS, N.; GRAVELL, A. M.; WILLS, G. B. Using Factor Analysis to Generate Clusters of Agile Practices (A Guide for Agile Process Improvement). In: AGILE Conference. Nashville, TN, USA: IEEE. 2010, p 11-20.

ABRAHAMSSON, P. et al. Agile Software Development Methods: review and analysis. VVT Publications. Espoo, Finland: 2002.

AGILEMANIFESTO. Manifesto for Agile Software Development. Disponível em: <http://www.agilemanifesto.org/>. Acesso em: 26 fev. 2016.

AMBLER, S. W. Agile Model Driven Development (AMDD): the key to scaling agile software development. Disponível em:

<http://www.agilemodeling.com/essays/amdd.htm>. Acesso em: 29 abr. 2015.

ASNAWI, A. L.; GRAVELL, A. M.; WILLS, G. B. Empirical Investigation on Agile Methods Usage: Issues Identified from Early Adopters. In: 12th International Conference XP. Madrid, Espanha. 2011, p 192-207.

AZIZYAN, G.; MAGARIAN, M. K.; KAJKO-MATSSON, M. Survey of Agile Tool Usage and Needs. In: AGILE Conference. Salt Lake City, UT, USA: IEEE. 2011, p 29-38. 
BARRETO, A. A. A questão da informação. Revista São Paulo em Perspectiva, v. 8, n. 4, 1994.

BECK, K. Extreme programming explained: embrace change. Boston: Addison-Wesley, 1999.

BOEHM, B.; TURNER, R. Balancing Agility and Discipline: a guide for the perplexed. Addison-Wesley Professional, 2004.

BOOCH, G.; RUMBAUGH, J.; JACOBSON, I. The Unified Modeling Language User Guide. 2. ed. USA: Addison-Wesley, 2005.

CARVALHO, L. A. C.; BARBOSA, M. W.; SILVA, V. B. Proposta e Avaliação de uma Abordagem Lúdica para o Ensino de Histórias de Usuário e Scrum.

Revista de Gestão e Projetos, v. 5, n. 3, p. 44-58, 2014.

$\mathrm{CHOO}, \mathrm{C} . \mathrm{W}$. Information management for the intelligent organization. Second edi ed. Medford, NJ: Information Today, Inc., 1998.

CHUA, A.Y.K.; YANG, C.C. The Shift towards Multi-Disciplinarity in Information Science. Journal of the American Society for Information Science and Technology, v. 59, n. 13, p. 2156-2170, 2008.

COCCO, L. et al. Simulating Kanban and Scrum vs. Waterfall with System Dynamics. In: 12th International Conference XP. Madrid, Spain. 2011, p 117131.

COCKBURN, A. Agile Software Development: the cooperative game. 2. ed. Boston: Pearson Education, Inc., 2007.

CODIAC, Y.F.L. Ciência da informação. Brasília: Briquet de Lemos, 1996.

CONBOY, K. Agility from first principles: reconstructing the concept of agility in information systems development. Information Systems Research, v. 20, n. 3, p. 329-354, 2009.

CONTADOR, J. L. et al. Sistema Kanban para fábrica de tintas. Revista de Administração e Inovação, v. 2, n. 1, p. 68-77, 2005.

DAVENPORT, T. H.; PRUSAK, L. Conhecimento empresarial. Rio de Janeiro: Campus, 2003.

DOLCI, D. B. Teorizando as práticas dos métodos ágeis no desenvolvimento de software visando ao processo de inovação das empresas. Teoria e Prática em Administração, v. 1, n. 1, p. 74-96, 2011. 
DORAIRAJ, S.; NOBLE, J.; MALIK, P. Effective Communication in Distributed Agile Software Development Teams. In: 12th International Conference XP. Madrid, Spain. 2011, p. 102-116.

DORAIRAJ, S.; NOBLE, J.; MALIK, P. Knowledge Management in Distributed Agile Software Development. In: AGILE Conference. Dallas, TX, USA: IEEE. 2012a, p 64-73.

DORAIRAJ, S.; NOBLE, J.; MALIK, P. Understanding Team Dynamics in Distributed Agile Software Development. In: 13th International Conference XP. Malmö, Sweden.2012b, p 47-61.

DORAIRAJ, S.; NOBLE, J.; MALIK, P. Understanding the Importance of Trust in Distributed Agile Projects: A Practical Perspective. In: 11th International Conference XP. Trondheim, Norway. 2010, p 172-177.

FERREIRA, J.; SHARP, H.; ROBINSON, H. Agile Development and User Experience Design Integration as an Ongoing Achievement in Practice. In: AGILE Conference. Dallas, TX, USA: IEEE. 2012, p 11-20.

FERREIRA, J.; SHARP, H.; ROBINSON, H. Values and Assumptions Shaping Agile Development and User Experience Design in Practice. In: International Conference on Agile Software Development XP. 2010, p 178-183.

FOWLER, M. The Agile Manifesto: where it came from and where it may go. Disponível em: <https://martinfowler.com/articles/agileStory.html>. Acesso em: 30 set. 2014.

GE, X.; PAIGE, R.F.; MCDERMID, J. An Iterative Approach for Development of SafetyCritical Software and Safety Arguments. In: AGILE Conference. Nashville, TN, USA: IEEE. 2010, p 35-43.

GHANAM, Y.; MAURER, F. Extreme Product Line Engineering-Refactoring for Variability: A Test-Driven Approach. In: 11th International Conference XP. Trondheim, Norway. 2010, p 43-57.

GRAEML, A. R.; CSILLAG, J. M. Customization in the manufacturing industry: survey results of a survey in Southeastern Brazil. Journal of Information Systems and Technology Management, v. 6, n. 3, p. 395-412, 2009.

GUIMARAES, L. F. A.; FALSARELLA, O. M. Uma análise da metodologia JustIn-Time e do sistema Kanban de produção sob o enfoque da ciência da informação. Perspectivas em Ciência da Informação, v. 13, n. 2, 2008. 
HIGHSMITH, J. Retiring Lifecycle Dinosaurs: using adaptive software development to meet the challenges of a high-speed, and high-change environment. Disponível em:

$<$ http://www.adaptativesd.com/articles/dinosaurs.pdf > . Acesso em: 29 abr. 2015.

HODA, R.; NOBLE, J.; MARSHALL, S. Agile Undercover: When Customers Don't Collaborate. In: 11th International Conference XP. Trondheim, Norway. 2010, p 73-87.

HODA, R.; NOBLE, J.; MARSHALL, S. Supporting Self-organizing Agile TeamsWhat's Senior Management Got to Do with It? In: 12th International Conference XP. Madrid, Spain. 2011, p 1-4.

JABBOUR, A. B. L. S. et al. Análise da relação entre manufatura enxuta e desempenho operacional de empresas do setor automotivo no Brasil. Revista de Administração, v. 48, n. 4, p. 843-856, 2013.

JUNIOR, M. L.; FILHO, M. G. Evolução e avaliação da utilização do Sistema Kanban em empresas paulistas. Revista de Administração, v. 44, n. 4, p. 380-395, 2009.

KAJKO-MATTSSON, M.; AZIZYAN, G.; MAGARIAN, M. K. Classes of Distributed Agile Development Problems. In: AGILE Conference. Nashville, TN, USA: IEEE. 2010, p 51-58.

KITCHENHAM, B. Procedures for performing Systematic Literature Reviews. Keele University Technical Report TR/SE-0401, ISSN:1353-7776, 2004.

KRUCHTEN, P. Introduction au Rational Unified Process. Editions Eyrolles, 1999.

KURAPATI, N.; MANYAM, V. S. C.; PETERSEN, K. Agile Software Development Practice Adoption Survey. In: 13th International Conference on Agile Software Development XP. 2012, p 16-30.

LARMAN, C. Agile and Iterative Development: a manager's guide. Boston: Addison-Wesley Professional, 2003.

LAUDON, K. C.; LAUDON, J. P. Sistemas de informações gerenciais. 11. ed. São Paulo: Pearson Education do Brasil, 2014.

$\mathrm{LI}, \mathrm{J}$ et al. Analysis of Federated and Global Scheduling for Parallel Real-Time Tasks. In: Euromicro Conference on Real-Time Systems (ECRTS). Madrid, Espanha: IEEE. 2014, p.85-96. 
LITTELL, J. H.; CORCORAN, J.; PILLAI, V. Systematic reviews and metaanalysis. New York: Oxford University Press, 2008.

MNKANDLA, E. A Selection Framework for Agile Methodology Practices: a family of methodologies approach. Johannesburg: University of The Witwatersrand, 2008. $247 \mathrm{f}$. Tese de Doctor of Philosophy - Faculty of Engineering and the Built Environment, University of The Witwatersrand, Johannesburg, 2008.

NEGREIRO, M.; BARBOSA, W. T. O problema de alocação de recursos e seleção de múltiplos projetos de TI. Revista de Gestão e Projetos, v. 4, n. 2, 2013.

NONAKA, I.; TAKEUCHI, H. The knowledge-creating company: how japanese companies create the dynamics of innovation. Oxford: Oxford University Press, 1995.

OECD. Oslo Manual: guidelines for collecting and interpreting innovation data. 3 ed. Paris: Author, 2005.

PALMER, S. R.; FELSING, J. M. A Practical Guide to Feature-driven Development. Prentice Hall PTR, 2002.

PEETERS, S. Applying Lean Thinking to Software Development. InfoQueue, Disponível em: <https://www.infoq.com/articles/applying-lean-thinking-tosoftware-development>. Acesso em: 4 mar. 2016.

PRESSMAN, R. S. Engenharia de Software: uma abordagem profissional. 7 ed. Porto Alegre: Bookman, 2006.

PRIES-HEJE, L.; PRIES-HEJE, J. Why Scrum Works: A Case Study from an Agile Distributed Project in Denmark and India. In: AGILE Conference. Salt Lake City, UT, USA: IEEE. 2011, p 20-28.

SARACEVIC, T. Information Science. Journal of the American Society for Information Science, v. 50, n. 12, p. 1051-1063, 1999.

SCHWABER, K. Scrum Development Process. In: SUTHERLAND J., CASANAVE C., MILLER J., PATEL P., HOLLOWELL G. (Orgs.). Business Object Design and Implementation. London: Springer, 1997.

SCHWABER, K.; BEEDLE, M. Agile Software Development with Scrum. 1 ed. Upper Saddle River, NJ: Pearson, 2001.

SCRUMSTUDY. A Guide to the SCRUM Body of Knowledge (SBOK GUIDE). Phoenix: VMEdu Inc., 2013. 
SELLITTO, M. A.; BORCHARDT, M.; PEREIRA, G. M. Assessing the degree of promptness of a service industry to adopt lean thinking. Revista Eletrônica de Administração, v. 9, n. 6, p. 1-20, 2003.

SENAPATHI, M.; SRINIVASAN, A. Understanding Post-Adoptive Agile Usage-An Exploratory Cross-Case Analysis. In: AGILE Conference. Salt Lake City, UT, USA: IEEE. 2011, p 117-126.

SILVA, R. E.; NETO, J. S. Contratação do desenvolvimento ágil de software na administração pública federal: riscos e ações mitigadoras. Revista do Serviço Público, v. 66, n. 1, p. 97-120, 2015.

SILVA, T.S. et al. User-Centered Design and Agile Methods: A Systematic Review. In: AGILE Conference. Salt Lake City, UT, USA: IEEE. 2011, p 7786.

SOARES, M. D. Metodologias Ágeis Extreme Programming e Scrum para o desenvolvimento de software. Revista Eletrônica de Sistemas de Informação, v. 3, n. 1, p. 1-8, 2004.

STAPEL, K. et al. Towards Understanding Communication Structure in Pair Programming. In: International Conference on Agile Software Development XP. 2010, p.117-131.

STAPLETON, J. DSDM, Dynamic Systems Development Method: the method in practice. Cambridge, Reino Unido: Addison-Wesley, 1997.

STETTINA, C. J.; HEIJSTEK, W.; FAEGRI, T. E. Documentation Work in Agile Teams: The Role of Documentation Formalism in Achieving a Sustainable Practice. In: AGILE Conference. Dallas, TX, USA: IEEE. 2012, p 31-40.

STEVENSON, W. J. Operations management. 10 ed. New York: MacGraw-Hill, 2009.

SUTHERLAND, J. Business Object Design and Implementation Workshop. In: 10th Annual Conference on Object-oriented Programming Systems, Languages, and Applications. Austin, TX, USA. 1995, p 170-175.

TAKEUCHI, H.; NONAKA, I. The new new product development game. Harvard Business Review, p. 137-147, 1986.

TERLIZZI, M. A.; BIANCOLINO, C. A. Projeto de Software no setor bancário: Scrum ou Modelo V. Tecnologias de Administração e Contabilidade, v. 4, n. 1, p. 46-58, 2014.

TOOLS4NOOBS. Online summarize tool. Disponível em: <https://www.tools4noobs.com/summarize/>. Acesso em: 3 dez. 2015. 
VALLERÃO, A. G.; ROSES, L. K. Monitoramento e controle de projetos de desenvolvimento de software com o Scrum: avaliação da produção científica. Revista de Gestão e Projetos, v. 4, n. 2, 2013.

VITORINO, C. M.; TERRA, L. D. B. The implementation of MRP II in two Brazilian industries. Revista Economia \& Gestão, v. 2, n. 4-5, p. 96-105, 2002.

WANG, X. The Combination of Agile and Lean in Software Development: An Experience Report Analysis. In: AGILE Conference. Salt Lake City, UT, USA: IEEE. 2011, p 1-9.

WILLIAMS, L.; RUBIN, K.; COHN, M. Driving Process Improvement via Comparative Agility Assessment. In: AGILE Conference. Nashville, TN, USA: IEEE. 2010, p 3-10.

\title{
Title
}

Agile Methods from the Viewpoint of Information

\begin{abstract}
:
Introduction: Since Paul M. G. Otlet highlighted the term documentation in 1934, proposing how to collect and organize the world's knowledge, many scientific researches directed observations to the study of Information Science. Methods and techniques have come up with a world view from the perspective of information. Agile methods follow this trend. Objective: The purpose is to analyze the relevance of information flow to organizations adopting agile methods, understanding how the innovation process is influenced by this practice. Methodology: This is a bibliometric study with fundamentals of Systematic Literature Review (SLR). The integration between the SLR technique interacting with Summarize tool is a new methodological proposal. Results: Scrum appears with the highest number of publications in SPELL. In comparison, results of Google Scholar pointed out to the importance of practices and team behaviors. In Science Direct repository, critical success factors in project management and software development are highlighted. Introduction:

Conclusions: It was evident that agile methods are being used as process innovations. The benefits and advantages are evident with internal and external occurrence of information flow. Due to the prevalence in the literature, Scrum deserves attention by firms.
\end{abstract}

Keywords: Agile methods. Process innovation. Information flow. Systematic Literature Review.

\section{Titulo}

Métodos Ágiles desde el Punto de Vista de la Información 


\section{Resumen:}

Introducción: Desde que Paul M. N. Outlet destacó el término documentación en 1934, proponiendo cómo recoger y organizar el conocimiento del mundo, muchas investigaciones científicas han dirigido sus esfuerzos al estudio de la Ciencia de la Información. Métodos y técnicas han llegado con una visión del mundo desde la perspectiva de la información. Los métodos ágiles siguen esta tendencia. Objetivo: La meta es analizar la relevancia del flujo de información para las organizaciones que adoptan los métodos ágiles, comprendiendo cómo el proceso de innovación va ser influenciado por esta práctica. Metodología: Este es un estudio bibliométrico con los fundamentos de la Revisión Sistemática de la Literatura (RSL). La integración entre la técnica RSL interactuando con la herramienta Summarize es una nueva propuesta metodológica. Resultados: El Scrum aparece con mayor número de publicaciones en SPELL. Los resultados de Google Scholar apuntaban para la importancia de las prácticas y comportamientos de los equipos. En comparación, el repositorio ScienceDirect, factores críticos de éxito en gestión y desarrollo de proyectos de software están resaltados. Conclusiones: Era evidente que los métodos ágiles están siendo utilizados como innovaciones de procesos. Los beneficios y ventajas son evidentes con la presencia interna y externa del flujo de información. Debido a la prevalencia en la literatura, el Scrum merece atención de las firmas.

Palabras clave: Métodos ágiles. Innovación de procesos. Flujo de información. Revisión Sistemática de la Literatura.

Recebido em: 16.04.2016

Aceito em: 12.12.2017 